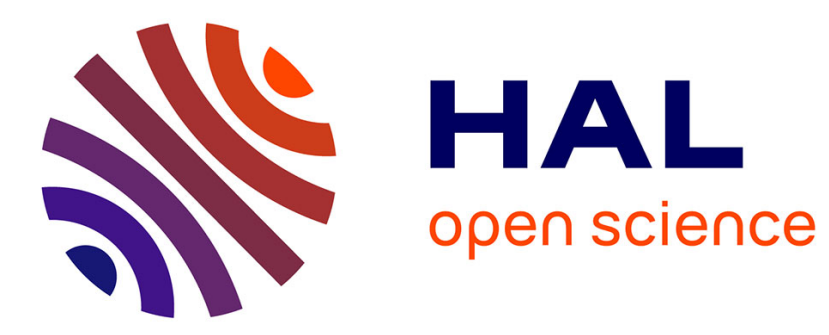

\title{
Errance du moi
}

Beatriz Carneiro dos Santos

\section{To cite this version:}

Beatriz Carneiro dos Santos. Errance du moi. Libres cahiers pour la psychanalyse, 2011, Grandeur et Solitude du Moi, 24 (2), http://www.cairn.info/revue-libres-cahiers-pour-la-psychanalyse-2011-2page-81.htm. 10.3917/lcpp.024.0081 . halshs-01490769

\section{HAL Id: halshs-01490769 \\ https://shs.hal.science/halshs-01490769}

Submitted on 15 Mar 2017

HAL is a multi-disciplinary open access archive for the deposit and dissemination of scientific research documents, whether they are published or not. The documents may come from teaching and research institutions in France or abroad, or from public or private research centers.
L'archive ouverte pluridisciplinaire HAL, est destinée au dépôt et à la diffusion de documents scientifiques de niveau recherche, publiés ou non, émanant des établissements d'enseignement et de recherche français ou étrangers, des laboratoires publics ou privés. 


\section{Errance du moi}

«JE SUIS NEE TANT ET TANT./ J'ai doublement souffert/dans la mémoire de l'ici et $d u$ delà », dit un poème d'Alejandra Pizarnik ${ }^{1}$. Poète argentine née en 1936 de parents immigrants juifs, elle est l'une des auteures les plus célèbres de l'Amérique Latine, connue à la fois par la valeur de son œuvre et par sa vie peu ordinaire de jeune artiste dans le milieu littéraire parisien des années 60 . Le petit poème par lequel j'initie ce travail indique un thème central de son écriture : l'errance, comprise comme un déplacement qui se fait en tous sens et sans se fixer nulle part. Les mouvements constants dont son écriture essaye de rendre compte inspirent une réflexion sur l'articulation mobile entre les identifications et le sentiment d'identité, et c'est ce que je propose de développer dans cet article.

L'écriture de Pizarnik, qu'elle soit destinée à devenir publique, tels ses poèmes (publiés dès 1955, lorsqu'elle avait 19 ans), ou qu'elle soit conçue comme communication privée (comme ses journaux et sa correspondance, rendus publiques après sa mort), est marquée par des images et des références à l'exil, au déplacement, au voyage, à l'errance, à une constante mobilité dans les espaces. Il ne s'agit pas uniquement d'une errance géographique, décrite dans poèmes et lettres composées dans différents pays, mais aussi de l'errance de l'exil de ses parents en Argentine(«Par mon sang juif, je suis une exilée. Par mon lieu de naissance, c'est à peine si je suis argentine (tout ce qui est argentin est irréel et diffus). Je n'ai pas de patrie $»^{2}$ ). Finalement, son œuvre parle aussi d'une errance parmi plusieurs temps, entre la vie de femme adulte et «l'enfant muette qui parle en [son] nom » ${ }^{3}$.

Dans le préface à Les travaux et les nuits, la traductrice S. B. Supervielle raconte comment, lorsque l'on lit Alejandra Pizarnik et que l'on se laisse habiter par sa présence « on revient perpétuellement au combat acharné avec les mots et leur signification, qui sont [pour Pizarnik] la parole introuvable, et perpétuellement à cette solitude absolue à laquelle on parle sans parvenir à se dire $»^{4}$. Ce combat et cette solitude orientent ses tentatives de trouver sa

\footnotetext{
${ }^{1}$ A. PIZARNIK (1962), «Arbre de Diane», in Les travaux et les nuits, traduction de S. B. Supervielle et C. Couffon, Granit/Unesco, 1986, p 38.

Sauf mention contraire, toutes les citations de Pizarnik sont extraites de ce recueil.

${ }^{2}$ A. PIZARNIK, Journaux (1959-1971), traduction de Anne Picard, José Corti, 2005

3 «Soumise à l'enfant muette qui parle en mon nom/je me ferme, me défends/ lorsque les choses/comme des troupes de trous/répondent à ma terreur », p. 271

${ }^{4}$ S. B. SUPERVIELLE, Préface à Les travaux et la nuit, op. cit. p.7-12
} 
voix et son silence : «Mais le silence est sûr. C'est pourquoi j'écris. Je suis seule et j'écris. Non, je ne suis pas seule. Il y a ici quelqu'un qui tremble $»^{5}$.

C'est à partir de ce tremblement qui accompagne Alejandra Pizarnik que je souhaite penser le mouvement créé par la présence de plusieurs identités dans ses poèmes. Dans ses écrits, cette présence n'est pas une succession de manières d'être, mais plutôt une construction à partir de la multiplicité même des voix qui meuvent un sentiment de soi auquel Pizarnik peut se référer. Autrement dit, la présence audible et simultanée de plusieurs voix à l'intérieur de son «moi propre", pour reprendre la formule de Freud ${ }^{6}$, peut faire trembler l'espace psychique dans lequel la poète se trouve. Cette polyphonie n'est jamais vécue comme plainte névrotique et encore moins comme une expérience psychotique; elle est plutôt une expérience, une construction qui participe au sens que Pizarnik attribue à son travail et à sa vie. Tel le souvenir à l'Acropole de Freud, qui lui fait percevoir deux voix qui « s'expriment » à l'intérieur de son $\mathrm{moi}^{7}$, le tremblement ressenti par Pizarnik suscite chez elle plutôt la curiosité que l'angoisse. Il semble ouvrir son sentiment d'identité à la pluralité et à la possibilité d'errer dans l'espace et dans le temps.

Chez Freud, le sentiment d'identité correspond à ce qu'il nomme, dans Le malaise dans la culture, le sentiment de soi. Il est défini comme une stabilité liée à la certitude d'un moi propre. Se reconnaître soi-même, ou plutôt se reconnaître en tant qu'un soi autonome, unitaire et bien démarqué de tout le reste, ${ }^{8}$ est l'exercice qui instaure la perception de l'identité propre. Le sentiment de soi et l'identité convergent dans la stabilité ou constance de traits identificateurs qui, alliée à l'unité (comprise comme la séparation qui certifie une existence dans une position ou état séparé) et au rapport de reconnaissance du même qu'elle rend possible, nous fait distinguables les uns des autres.

Ce sentiment de soi semble être toujours en mouvement dans l'œuvre de Pizarnik. Ses poèmes sont propulsés par la tentative, à chaque fois déçue mais toujours renouvelée, de trouver (ou retrouver) ce qui serait le mot, la poésie, la voix, le sens d'exister « au-delà de

\footnotetext{
${ }^{5}$ p. 155.

${ }^{6}$ Voir S. FREUD (1929[1930a], « Le malaise dans la culture », in Euvres Complètes, t. XVIII, Paris, PUF, $1994, p ; 251$

7 Face à la vision de l'Acropole, Freud se rend compte d'une double opération mentale : il y une voix qui exclame : «ainsi donc tout cela existe effectivement comme nous l'avons appris à l'école?!» et une voix qui perçoit cette déclaration L'une se surprend de l'existence même de quelque chose, l'autre observe cela même que la première énonce. Voir S. FREUD (1936[1936a]), « Lettre à Romain Rolland (un trouble du souvenir sur l'Acropole), in Euvres Complètes, vol. XIX, Paris, PUF, 2004, p. 331

${ }^{8}$ S. FREUD (1929[1930a)], op. cit., p. 251
} 
[soi]-même, comme une idée fixe $»^{9}$. Le parcours de la construction identitaire devient dans ses compositions une trajectoire sans destinée déterminée, mais plutôt composée d'oscillations entre des positions multiples : «tout en moi parle avec son ombrelet chaque ombre avec son double $»^{10}$. Ce parcours est loin de toute tentative d'associer l'identité à une détermination rigide de ce qu'est un sujet. Au contraire, son langage, avec les déplacements dont il essaie inlassablement de rendre compte, évoque une construction de soi qui ne cesse de se faire transformable. Et l'identité y fait apparition comme ce qui, tout en étant doté d'une unité suffisamment reconnaissable, s'ouvre à un monde polyphonique : "Je ne peux parler avec ma voix mais avec mes voix $\gg{ }^{11}$.

La construction d'un sentiment d'identité basé sur une trajectoire errante est repérable notamment dans les références que Pizarnik fait au temps de l'enfance. Très souvent, ses poèmes expriment, au présent, la liaison existante entre la poète adulte et une voix venue de l'enfance. L'Alejandra qui écrit est une femme et, en même temps, une «fillette de craie $\operatorname{rose}^{12} »$. Elle est entre le passé et le présent; son identité circule sans se fixer dans une temporalité correspondante à une détermination chronologique. Avec ses mots, elle est constamment en train de jouer avec « ce temps qui ne passe pas ${ }^{13} »$. Sa poésie lui permet de transformer le rapport avec le temps et rend possible des paradoxes tels que la nostalgie de la vieillesse ou la maturité de la petite enfance : «Je me souviens de mon enfancelquand j'étais une vielle dame $»^{14}$.

Ce rapport particulier au temps présent dans les travaux de Pizarnik, ce mouvement constant qu'elle décrit d'une position subjective à une autre, fait penser à l'articulation entre les liens d'identification constitués dans l'enfance et les transformations identitaires passibles d'avoir lieu à l'âge adulte. Plus précisément, l'écriture de Pizarnik, avec le jeu qu'elle installe entre les vécus infantiles (dont la « voix » reste toujours audible) et l'errance permanente du sentiment de soi, m'invite à relire la théorisation des identifications à travers le prisme de sa temporalité multiple. Je souhaite établir comment l'expansion de l'appareil notionnel psychanalytique transforme constamment le concept d'identification. Il s'agit, en même temps, de souligner comment cette transformation opère sur la notion de moi et accorde chaque

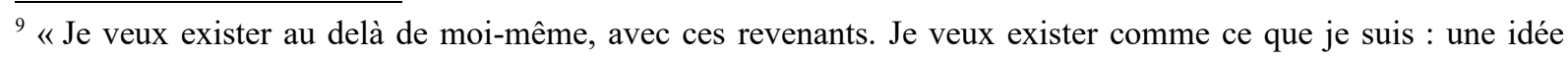
fixe. Je veux aboyer, non louer le silence de l'espace duquel on naît », p. 237

${ }^{10}$ p. 227

${ }^{11}$ p. 174

${ }_{12}^{12}$ « Comme une fillette de craie rose sur un mur très vieux subitement effacée par la pluie », p. 154.

${ }^{13}$ Titre d'un travail de J.B. PONTALIS, Ce temps qui ne passe pas, Paris, Gallimard, 1997

${ }^{14}$ p. 54
} 
théorisation de l'identification avec une théorisation du moi. Dans ce travail, l'errance de Pizarnik accompagne, tel un deuxième instrument de musique qui accompagne une mélodie, l'avancement méthodique de Freud vers une conception de l'identification. Cet accompagnement se fait dissonant parfois pour bien indiquer qu'il a lieu entre une écriture poétique et l'écriture analytique freudienne, orientée par la rigueur de sa méthode. Et dans cette dissonance il indique encore un thème freudien, à savoir le rapport particulier qui peut exister entre les artistes et la psychanalyse.

Lorsque Freud présente une énumération des types d'identification qui, jusque là, se trouvaient diffus dans différents textes, les changements dans le concept sont clairs. Il s'agit du chapitre VII de Psychologie des masses et analyse du moi, nommé «L 'identification ». Dans ce texte, nous suivons l'évolution de la fonction de l'identification, qui passe de l'idée d'identification comme une défense à l'idée de l'identification comme organisation dotée d'une position changeante dans la théorie de la formation du moi.

Nous y voyons comment, au départ, les apparitions de la notion d'identification sont proches du verbe identifier et de l'idée de «reconnaître pour identique »: la relation d'identification entre deux termes suppose soit que l'un se substitue à l'autre, soit que l'un sert à identifier l'autre ${ }^{15}$. Elle est pensée à partir de la conception d'un lien direct entre le moi et la conscience (celui-là étant un «territoire » plus vaste que celle-ci). Lorsque Freud parle de l'identification que certaines de ses patientes peuvent avoir avec les « femmes de basse moralité $\gg^{16}$, ou quand il décrit les symptômes hystériques de Dora ${ }^{17}$, il se réfère à un fonctionnement qui sert à évacuer les contenus perturbateurs à l'œuvre dans l'appareil psychique. L'identification y est décrite comme une sorte d'action défensive qui fournit aux sujets une possibilité (symptomatique) de supporter l'impact d'un contact déplaisant et n'est donc pas une partie constitutive du moi.

Plus tard, elle décrit un processus plus complexe. Freud signale que : «la recherche psychanalytique, qui, à l'occasion, s'est déjà aussi attaquée aux problèmes plus difficiles des psychoses, a pu nous

\footnotetext{
${ }^{15}$ S. FREUD (1899(1900a)], « L'interprétation du rêve», in Euvres Complètes, t. IV, Paris, PUF, 2004 ${ }^{16}$ S. FREUD (1897), « Lettre à Fliess du 2-5-1897 », in La naissance de la psychanalyse, Paris, PUF, 1996

${ }^{17}$ Nous pensons notamment à la toux, symptôme "partagé » avec le père, et à l'irritation de la gorge, « semblable » à celle de Mme. K. voir S. FREUD (1901[1905e]), « Fragments d'une analyse d'hystérie (Dora)», in Cinq psychanalyse s, Paris, PUF, 1970
} 
faire voir aussi l'identification dans quelques autres cas qui ne sont pas immédiatement accessibles à notre compréhension ${ }^{18}$ 》.

Il se réfère à l'identification homosexuelle et à l'identification mélancolique, et à la question qu'elles suscitent concernant l'introjection de l'objet dans le moi et la transformation moïque qui peut en découler.

Plus précisément, lorsque Freud formule la théorie du narcissisme et conclut que les mouvements pulsionnels ont lieu dans et à partir du corps, par étayage avec les fonctions biologiques et par l'expérience de l'amour narcissique, le rôle de l'identification est aussi reformulé. Il devient identification par la personne aimée au lieu d'identification à cette personne $^{19}$, et porte maintenant la marque de la mélancolie : le moi s'identifie à l'objet d'amour abandonné dans un processus mélancolique qui lui permet de conserver son rapport à la personne aimée. Comme conséquence, un lien est établi entre la détermination (aussi mouvante soit-elle) de l'hétérosexualité et de l'homosexualité, et Freud énonce l'existence d'une identification homosexuelle « aux traits d'une régression narcissique $»^{20}$, en opposition au choix objectal qui se fait sous le modèle de l'amour à la mère.

Finalement, la dernière théorisation de l'identification la place au centre du carrefour œdipien, entre l'amour pour l'un des parents et hostilité envers l'autre, mais aussi entre l'amour et la haine à l'égard de chacun des parents. A partir de la formulation de la deuxième topique et de la transformation de la conception de moi (centrée maintenant sur son aspect clivé, distinct de la conscience), l'identification se transforme et fait désormais partie de la problématique de l'idéal du moi. Le complexe d'Edipe est maintenant ${ }^{21}$ décrit en sa forme « complète », en opposition au complexe simple et positif. Les destins œdipiens dits simples, unidirectionnels, forment une image qui n'est plus jalonnée par deux positions précises (amour pour l'un des parents, hostilité envers l'autre), mais est plutôt celle d'une «constellation œdipienne», selon les mots du psychanalyste belge Jean Florence ${ }^{22}$. L'identification au père se fait avec le maintien de la mère comme objet d'amour du complexe positif, et comme substitution de l'objet paternel du complexe négatif.

Le chapitre VII de Psychologie des masses et analyse du moi, avec l'énonciation des différentes fonctions de l'identification qu'elle présente, nous permet de repérer dans l'œuvre

\footnotetext{
${ }^{18}$ S. FREUD (1922[1922d)], « Le moi et le ça », in CEuvres Complètes, t. XVI, Paris, PUF, 1991, p. 46

${ }^{19}$ J. LAPLANCHE aborde cette question dans Sexual. La sexualité élargie au sens freudien, Paris, PUF

${ }^{20}$ S. FREUD (1910), Un souvenir d'enfance de Leonard da Vinci, Paris, Gallimard, 1987

${ }^{21}$ Voir S. FREUD (1922[1923b]), op. cit.

22 J. FLORENCE (1978), L'identification dans la théorie freudienne, Bruxelles, Facultés Universitaires Saint Louis, 1978
} 
de Freud le passage de l'identification, au singulier, à des identifications, plurielles, multiples et dirigées à plus d'un objet ou d'une direction. Ce faisant, il lance les fondations pour la formulation de l'identification primaire (qui a lieu un an plus tard, dans Le moi et le ça) et pour le renversement d'une chronologie basée sur les étapes du développement que celle-ci préconise. En effet, la description d'une identification qui est plus précoce que tout investissement et qui s'associe au père de la "préhistoire personnelle », instaure un temps entre l'auto-érotisme et l'amour du choix d'objet qui rend contre-intuitive la perception d'un « développement» des processus identificatoires. Comme le signale Jean Florence, en tant que « la première et la plus importante identification de l'individu $»^{23}$, elle continue d'exercer sur les identifications ultérieures une force d'attraction ou captation, tout en servant de « dispositif d'écoulement [ablauf]» à ceux-ci. En effet, l'identification primaire, tout en ayant lieu avant le temps de l'Edipe, ne prend tout son sens qu'avec l'instauration du complexe œdipien. La préhistoire primaire a besoin de l'histoire œdipienne pour se rendre intelligible de manière rétroactive.

Cette temporalité particulière des identifications fait partie de la mise en mouvement à l'œuvre dans ce que j'ai appelé errance. L'image d'un déplacement que l'errance évoque résonne avec les modifications qui ont eu lieu au long de la formulation du concept d'identification. L'errance à l'intérieur de la théorie, entre l'hystérie et la problématique de l'homo/hétérosexualité, rend visible la transformabilité de ce qui configure un sentiment de soi. Parallèlement, elle souligne l'importance foncière de la liaison entre les enjeux œdipiens et la constitution d'une identité par l'adulte, ce qui n'est pas sans intérêt pour les discussions sur les cliniques actuelles (je pense par exemple au thème de identités sexuelles). L'errance des identifications, entre le sillage du "parfum d'oiseau caressé » de l'enfance, toujours perceptible, et «la main qui tire vers l'autre rive», pour reprendre les mots d'Alejandra Pizarnik $^{24}$, nous permet de penser la problématique de la construction de l'identité sans nous figer dans une « actualité » de la vie adulte. Elle nous éclaircit sur les enjeux de la perception d'un sentiment de soi en considérant à la fois l'impact des vécus œdipiens et l'ouverture aux différentes voix qui nous constituent.

«L'errance, la chanson de nous deux, je tremble comme dans une métaphore l'âme comparée à une chandelle $»^{25}$. Dans l'écriture de Pizarnik, les mouvements constitutifs d'un

\footnotetext{
${ }^{23} \mathrm{idem}$

${ }^{24}$ "Je ne sais de l'enfance/qu'une peur lumineuse/une main qui m'entraîne/vers mon autre rive./mon enfance/et son parfum d'oiseau caressé », in A. PIZARNIK (2007), Poesia (1955-1972), Buenos Aires, Lumen, p. 325 ${ }^{25}$ p. 233
} 
devenir sujet se font perceptibles en tant que tels : mouvements, errances, tremblements. Néanmoins, l'enfance reste un point de repère, source abondante de matériaux à son œuvre. Tel un tissu, décrit par l'artiste Patrice Hugues comme étant « d'une part la rigueur, à l'opposé, l'imprévisible $»^{26}$, le sentiment de soi chez Alejandra Pizarnik n'est ni figé ni entièrement à construire. Il est d'un côté la rigueur du compte de fils en trame et chaîne et de l'autre son inverse, « les plis imprévisibles qui se forment à chaque mouvement et selon chacun de ses positionnements ». D'un côté l'unité et la constance des repères organisés dans et par l'enfance, de l'autre la multiplicité mobile de traits subjectifs qui appartiennent à (et aussi constituent) la part du moi qu'elle reconnaît comme soi. L'écriture de l'errance de Pizarnik nous rend sensibles au " point mobile fixe » sur lequel nous nous construisons et, ce faisant, nous inspire à être à l'écoute des mouvements, plus qu'à des positions.

Beatriz CARNEIRO DOS SANTOS, psychologue, docteur à l'Ecole Doctorale Recherches en Psychanalyse (Université Paris Diderot)

\footnotetext{
${ }^{26}$ P. HUGUES (2005), « entre-deux », in : P. Hugues et R.Debray, Dictionnaire culturel du tissu, Babylone/Fayard, Lyon, p.94.
} 